\title{
Motorola versus radioastronomy
}

SIR - I should like to add to Alison Abbott's News item, "Mobile telephones ring out changes for radioastronomy frequencies" (Nature 380, 569; 1996), in which she reports on the technical conflict between Motorola's proposed Iridium global mobile telephone system and the radioastronomy community about the jamming Iridium is likely to cause in the $1,610.6-1,613.8 \mathrm{MHz}$ radioastronomy band.

Motorola, in designing the Iridium system, chose to use the upper part of the 1,613.8-1,626.5 $\mathrm{MHz}$ band for the downlinks from their low-Earth-orbit satellites to their individual hand-held 'subscriber' mobile telephones. They are entitled to do this because the band is allocated by the International Telecommunication Union (ITU) to the Mobile-Satellite Service (MSS). But the allocation for use in the space-to-Earth direction is termed 'secondary', which means the MSS must accept interference from any service having a 'primary' allocation in the same band and it must itself, of course, not cause interference to a primary service.

Unfortunately for Motorola, the Radio Astronomy Service (RAS) has a primary allocation in the neighbouring band of 1,610.6-1,613.8 MHz, which embraces one of the spectral lines of the $\mathrm{OH}$ radical, and radiotelescopes are peculiarly susceptible to interference from transmissions from satellites.

To communicate directly with hand-held telephones, the Iridium satellites need to transmit an amount of power which is sufficiently significant, in terms of what is available on a satellite, for it to be necessary to use high-efficiency radio-frequency power amplifiers. But it is an unfortunate truth that all high-efficiency power amplifiers are nonlinear. This would not matter if the signal to be transmitted were of constant amplitude or in the nature of pulses, only on or off. But such an amplifier inevitably distorts a signal that varies in amplitude. Now the system architecture chosen by Motorola requires the amplifiers to handle simultaneously several independent radio-frequency signals aimed at different subscribers. Even though each signal on its own would not suffer distortion, the superposition of several, each on its own 'carrier frequency', creates a composite signal that varies in amplitude. This inevitably leads to 'intermodulation distortion' - spurious frequency components are generated both inside and outside the band occupied by the intended signals.

This would still not matter if the satellites each had only one power amplifier, as it would then be possible for it to be followed by a suitable output filter to remove at least the 'out-of-band' spurious frequency components. But Motorola has further chosen to use 'phased arrays' on the satellites - no less than seven arrays per satellite - and each array is composed of a large number of individual amplifiers. To equip them all with adequate output filters is difficult and would add, perhaps prohibitively, to the weight of the satellites.

The power in the intermodulation products falling in the RAS band would depend on the amount of telephone traffic being carried by Iridium and is hard to estimate with any accuracy. Typically it might be a thousand times above the level acceptable to the RAS and it therefore would amount to total jamming of the band from the point of view of astronomical research.

Motorola should have recognized at an early stage of its system design that it would be impossible to build phased arrays of power amplifiers that meet the requirements of the Radio Regulations of the ITU; these state clearly that the RAS is primary and the MSS downlink is secondary, and a special blanket footnote (FN733E) specifies that "[h]armful interference shall not be caused to stations of the radio astronomy service using the band 1,610.6-1,613.8 $\mathrm{MHz}$ by stations of the radiodeterminationsatellite and mobile-satellite services".

Motorola has stubbornly persisted with its defective system architecture. Instead of going back to the drawing-board and designing a system to higher engineering standards, it has become intent on forcing the radioastronomy community to accept curtailment of its ability to make observations in this band.

It is not clear how this conflict will end, but if Iridium goes ahead as planned it will be piracy in the radio spectrum, it will make a mockery of the ITU and its Radio Regulations, and it will be a disaster for radioastronomy.

\section{John Ponsonby}

Onsala Space Observatory,

S-439 92 Onsala, Sweden

\section{Citation policy on sequences}

SIR - Although editorial boards of scientific journals strive to communicate as objectively and as rigorously as possible, there is an important issue that has not been dealt with by these boards. A survey of the 'Instructions to Authors' from a variety of international journals, including Nature and Science, indicates no clear policy on the citation of sequence information. The policy may dramatically influence the measurement of both the citation index and journal impact factors (JIFs), given the proliferation of complete-genome sequencing projects.

When citing sequence information generated by other laboratories, an author has two seemingly equivalent options; accession numbers may be quoted, or the manuscript in which the sequence was reported may be cited. As both sources are increasingly cross-referenced, the former citation may be preferred by some authors; the data will be more immediately accessible, while the original sequence data decreasingly appears in the manuscript. But the two options have drastically different effects on the sequence generator's citation index, as well as the JIF of the journal in which the original article appeared. Although it can be argued that the sequence itself is not particularly scientifically meritorious, the same criticism could easily be levelled at method papers that have nevertheless enjoyed huge citation indices; scientists simply learn to distinguish between a muchcited method paper and a much-cited paper that makes central advances in a particular field. The lack of a formal policy means that citation indices and JIFs are fuzzier measurements than are presently appreciated. The simplest policy solution is that sequences should always be cited by both publication and accession number.

A related issue concerns the assignment of priority to a particular piece of sequence data. For example, the $16 \mathrm{~S}$ ribosomal RNA gene from the honeybee mitochondrion was originally sequenced by Vlasak et al. ${ }^{1}$. Has the appropriate citation been superseded by the publication of the complete sequence of the honeybee mitochondrial genome ${ }^{2}$, or do the original authors have priority? The distinction is an important one for editorial boards to consider, given the increase in complete-genome sequencing projects in recent years. It seems clear that history should have precedent, unless the original data are found to be flawed. But until editorial boards clarify this issue, citations will continue to be misappropriated.

Mark Dowton

Department of Crop Protection,

Waite Campus,

Adelaide University,

Glen Osmond, 5064, Australia

and

Department of Biology,

Wollongong University,

Wollongong 2522, Australia

1. Vlasak, I., Burgschwaiger, S. \& Kreil, G. Nucleic Acids Res. 15, 2388 (1987).

2. Crozier, R. H. \& Crozier, Y. C. Genetics $133,97-117$ (1993).

\section{corres@nature.com}

Letters submitted for Correspondence should be typed, double-spaced, on one side of the paper only, or emailed (with full mailing address) to corres@nature.com 